\title{
Evaluación de la transferencia de adolescentes desde una Unidad de Cardiología Pediátrica a una de Adultos
}

\author{
Pamela Oliva ${ }^{1}$, Michael Soto ${ }^{2}$, Juan Orellana $^{3}$, Pedro Véliz $^{4}$, María Isabel Toledo ${ }^{1}$, Constanza López ${ }^{5}$, Francisca Bonilla ${ }^{4}$ \\ 1 Cardióloga Infantil, Hospital Dr. Hernán Henríquez Aravena de Temuco, Universidad de la Frontera (UFRO). \\ 2 Interno Medicina, UFRO. \\ 3 PhD Salud Pública, Facultad de Medicina, UFRO. \\ 4 Becado Pediatría, UFRO. \\ 5 E.U. Cardiología Infantil, Hospital Dr. Hernán Henríquez Aravena de Temuco.
}

Objetivos: evaluar la transferencia de adolescentes desde Cardiología Pediátrica (CP) a Cardiología de Adultos (CA) en el Hospital Dr. Hernán Henríquez de Temuco (HHHT), determinando si recibieron su primera atención en CA en la fecha requerida y si los controles se ajustaron al plazo indicado. Evaluar si la complejidad de la cardiopatía y ruralidad se asocian al cumplimiento en ambos objetivos, en los pacientes sin seguimiento adecuado.

Material y Método: revisión de fichas de pacientes atendidos en CP del HHHT que, cumplida la edad requerida, fueron transferidos a $\mathrm{CA}$, entre Octubre 2014 - Julio de 2017.

Resultados: De 53 pacientes transferidos de CPCA, 12 (22.6\%) tuvo traspaso no efectivo; de ellos, 6 presentaban complejidad moderada-alta $(66,6 \%)$ y 3 eran rurales $(25 \%)$. De 28 pacientes ingresados a CA, $14(50 \%)$ tenían seguimiento inefectivo; de ellos 13
(92.6\%) tenían cardiopatías de moderada-alta complejidad y $3(21.5 \%)$ provenían de áreas rurales.

Conclusiones: la transferencia de CP - CA fue inefectiva en $22.6 \%$ de los pacientes; la complejidad de la cardiopatía no se asoció al resultado de la transferencia en este grupo. En CA el seguimiento fue inefectivo en la mitad de los pacientes; de ellos, $92.8 \%$ tenían cardiopatías de moderada-alta complejidad, lo que fue estadísticamente significativo respecto de la complejidad en todos los pacientes traspasados. El domicilio no estuvo asociado a la pérdida de pacientes en la transferencia ni en el seguimiento. Estos hallazgos nos permitieron tomar medidas de mejoría en la transferencia y los controles en CA que esperamos mejoren los resultados futuros.

Palabras claves: Cardiopatía congénita en el adulto; transferencia de cuidados; cardiopatías congénitas; adolescencia. 


\section{Transfering adolescents from pediatric to adult cardiology units: evaluation.}

Aim: To evaluate the transfer of adolescents from Pediatric Cardiology (PC) to Adult Cardiology (AC) at Hospital Dr. Hernán Henríquez de Temuco (HHHT). We determined whether they got their first care in AC on the required date and whether controls were performed on the period prescribed. Also, to evaluate whether the complexity of cardiac disease and the patient's place of residence influenced the accomplishment of the objectives, in patients without adequate following. Material and Method: Retrospective review of clinical records of patients that were treated in PC at HHHT who, having the required age were transferred to AC, between October 2014 and July 2017.

Results: Of 53 patients that were transferred from $\mathrm{PC}$ to $\mathrm{AC}, 12(22.6 \%)$ had an non effective transfer; 6 of them had moderate to high complexity cardiac lesions $(66.6 \%)$ and 3 lived in rural areas (25\%). Of
28 patients admitted to AC, 14 (50\%) had a non effective follow up and 13 (92.8\%) had moderate to high complexity forms of heart disease; $3(21.5 \%)$ came from rural areas.

Conclusions: Transfer of patients from PC to AC was not effective in $22.6 \%$ of patients. The complexity of the disease was not related to the results of transfer in this group. At AC the follow-up was not effective in half the patients; from these, $92.6 \%$ had moderate to high complexity heart disease, which was significantly different from the complexity of all patients transferred. The place of residence was not relates to transfer neither to follow-up. These findings allowed us to make improvement in the transfer processes and the follow up at AC.

Keywords: Congenital heart disease in the adult; patient transfer; adolescence. 


\section{Introducción:}

La transición y transferencia ${ }^{1,2}$ de adolescentes con enfermedades crónicas desde Servicios Pediátricos a Adultos, son tareas complejas que deben abordar todos los profesionales que trabajan en las subespecialidades pediátricas. La transición es definida como el proceso por el cual los adolescentes y adultos jóvenes con enfermedades crónicas de la infancia, son preparados para hacerse cargo de sus vidas y salud en la adultez. La transferencia se refiere al evento o serie de eventos donde la responsabilidad del paciente se pasa a un proveedor de adultos. En el área cardiológica, los estudios muestran que el 85-90\% de los niños con Cardiopatías Congénitas (CC) sobreviven a la edad adulta ${ }^{3,4,5}$, que gran parte de ellos requieren seguimiento de por vida, aún después de realizados sus tratamientos intervencionales o quirúrgicos ${ }^{2,3}$, y que hay falta de seguimiento o lapsos entre el $7-76 \%$ de los pacientes transferidos a la atención de adultos ${ }^{6,7}$. Esta falta de seguimiento se ha reflejado de tal manera que en EEUU menos del 30\% de los adultos con CC se controlan en Centros especializados y de estos, menos del 15\% tienen cardiopatías clasificadas como severas; sin embargo, el 50\% de los pacientes adultos con CC tendrían lesiones complejas ${ }^{1}$. En la actualidad, en países desarrollados el número de pacientes adultos con CC supera al de niños y adolescentes portadores de ellas. En Canadá se estimó que dos tercios de la población con CC era adulta ${ }^{8}$ y otro estudio mostró que esta población crece un $5 \%$ anual $^{3}$. Las dificultades para llevar a cabo con éxito los procesos de transferencia y seguimiento de los pacientes adolescentes portadores de $\mathrm{CC}$ a la atención de adultos es un problema mundial y conlleva riesgos significativos para la calidad de vida y sobrevida de ellos. En Chile esta realidad se vive en forma evidente, agravada por el hecho de que no hay suficientes Cardiólogos de Adultos preparados en el manejo de pacientes portadores de CC y tampoco hay suficiente cantidad de Cardiólogos Pediátricos con conocimiento de las cardiopatías del adulto. Existe sólo un Centro Cardioquirúrgico en el Sistema Público para atender a todos los pacientes transferidos; tampoco hay un programa formal de formación de especialistas en esta área y no todos los Becados de CA tienen establecido dentro su formación rotaciones por Centros especializados en Cardiopatías Congénitas del Adulto. En nuestro Hospital existe Cardiocirugía de Adultos desde 1989, Cardiología Pediátrica desde 1991 y un Cardiólogo Adulto con formación en Cardiopatías Congénitas desde 2003.

\section{Objetivos:}

Evaluar la efectividad de la transferencia de adolescentes portadores de CC desde la atención de CP a la de adultos en el HHHT, determinando si recibieron su primera atención en CA en la fecha requerida medicamente, y si después de ella los controles siguientes se ajustaron a lo indicado. Hay que señalar que a la fecha no existe un programa de transición formal, pero si una transferencia protegida dentro del mismo Centro (horas reservadas a través de oficina de admisión para la transferencia y controles posteriores en policlínico de CC del Adulto), que la atención se realiza en un mismo edificio y que se comparten las mismas fichas clínicas. Se evaluó, además, si el domicilio (ruralidad) del paciente y la complejidad de la cardiopatía se asocian al cumplimiento en los pacientes sin seguimiento adecuado.

\section{Material y método:}

Se revisaron las fichas clínicas de los pacientes atendidos en la Unidad de CP del HHHT que, cumplida la edad requerida (15-18 años), fueron transferidos a la atención de Cardiopatías Congénitas del Adulto, entre Octubre de 2014 y Julio de 2017. La fecha del primer control en CA la determinó el Cardiólogo Infantil de acuerdo con el estado clínico del paciente y la complejidad de su cardiopatía.

Dado que en la literatura no existe un consenso respecto de los criterios para un buen cumplimiento de la transferencia y del seguimiento posterior, se establecieron como criterios locales los siguientes:

- Control al día: control efectuado en CA en la fecha correspondiente y con rango de hasta $50 \%$ y tope de 6 meses, posterior al tiempo de citación.

- Control fuera de plazo: el efectuado en CA entre el 51\% y hasta el $100 \%$ de tiempo posterior al tiempo de citación, con tope de 1 año.

- Paciente perdido: el que no acudió al control a CA después del 100\% del tiempo de citación, o más de un año del plazo requerido.

- Control pendiente dentro de plazo: pacientes que aún no les corresponde control en CA respecto de la fecha de citación.

- Control incompleto: paciente evaluado en CA, con exámenes solicitados, pero pendientes de realizar por más 
de 3 meses.

- Control antes de plazo: control en CA antes del 50\% del plazo de citación.

Respecto del domicilio, se definió como Rural a las áreas no urbanas de la Región y Urbano a otras ciudades de la Región. Se identifica a Temuco como a la ciudad en su zona urbana, particularmente por ser el lugar donde se ubica el Hospital que realiza la atención especializada de los pacientes en estudio.

La complejidad de la cardiopatía fue determinada con los Criterios de Bethesta ${ }^{9}$, modificados por la definición local de que los pacientes portadores de marcapasos y Tetralogía de Fallot ${ }^{8}$ son considerados de alta complejidad, ya que tienen riesgos mayores, requieren atención cardiológica por profesionales con experiencia en esas patologías y equipamiento especializado para sus evaluaciones.

\section{Resultados:}

Efectividad de la transferencia: Durante el período del estudio, se transfirieron 53 pacientes desde CP a CA. De ellos, 26 (49.1\%) tenían cardiopatías congénitas de complejidad leve, $16(30.2 \%)$ moderada, 11 (20.7\%) alta. Hubo 2 casos de jóvenes que no tenían cardiopatía, pero estaban en control por enfermedad sistémica o antecedente familiar con riesgo cardiovascular, los que para el efecto del estudio se incorporaron como leves. En cuanto al domicilio, 16 (30.2\%) eran de Temuco, 22 (41.5\%) urbanos y $15(28.3 \%)$ rurales. Respecto de la efectividad de la transferencia, en 17 casos $(32.1 \%)$ el primer control fue efectuado al día en Cardiopatías Congénitas del Adulto y en $5(9.4 \%)$ antes de plazo, considerándose ambas situaciones como buen cumplimiento (total 41.5\%). En 19 adolescentes (35.9\%) la atención estaba pendiente dentro de plazo, por lo que aún no les correspondía control. En 6 pacientes (11.3\%) el control ocurrió fuera de plazo y otros $6(11.3 \%)$ estaban perdidos, considerándose ambas condiciones como transferencia inefectiva (total $22.6 \%$ ). De los 12 adolescentes con transferencia inefectiva, 4 tenían cardiopatías de complejidad leve (33.3\%) y 8 moderada-alta $(66.7 \%)$, lo que no fue estadísticamente significativo respecto del grupo total de 53 pacientes trasladados $(p=0.182$, test exacto de Fisher). En cuanto al domicilio, 3 procedían de áreas rurales (25\%) y 9 de zonas urbanas y Temuco $(75 \%)$, lo que tampoco tuvo significación estadística respecto del grupo total de 53 pacientes ( $\mathrm{p}=0.843$ test exacto de Fisher). (Tabla 1).

\begin{tabular}{l|l|l|}
$\begin{array}{l}\text { Tabla 1: } \\
\text { Efectividad de la transferencia de Cardiología Pediátrica a } \\
\text { Cardiología Adultos. }\end{array}$ & \begin{tabular}{l} 
No Pacientes \\
\hline
\end{tabular} & $\%$ \\
\hline Control al día & 17 & 32,1 \\
\hline Control antes de plazo & 5 & 9,4 \\
\hline Control fuera de plazo & 6 & 11,3 \\
\hline Pacientes perdidos & 6 & 11,3 \\
\hline Control pendiente dentro plazo & 19 & 35,9 \\
\hline Total & 53 & 100 \\
\hline
\end{tabular}

Control al día: control efectuado en CA en la fecha correspondiente y con rango de hasta $50 \%$ y tope de 6 meses, posterior al tiempo de citación.

Control fuera de plazo: el efectuado en CA entre el $51 \%$ y hasta el $100 \%$ de tiempo posterior al tiempo de citación, con tope de 1 año.

Paciente perdido: el que no acudió al control a CA después del $100 \%$ del tiempo de citación, o más de un año del plazo requerido.

Control pendiente dentro de plazo: pacientes que aún no les corresponde control en CA respecto de la fecha de citación.

Control antes de plazo: control en CA antes del 50\% del plazo de citación.

Efectividad del seguimiento en CA: de los 28 pacientes que ya habían ingresado al Policlínico de Cardiopatías Congénitas del Adulto, 9 (32.1\%) llevaban controles de seguimiento al día y 1 (3.6\%) antes de plazo, por ello considerados con buen seguimiento (total $35.7 \%$ ). En 4 casos $(14.3 \%)$ los controles estaban pendientes dentro de plazo. Sin embargo 5 (17.9\%) llevaban control incompleto y $9(32.1 \%)$ estaban perdidos, lo cual se evaluó como seguimiento inefectivo (total 50\%) (Tabla 2). De estos 14 pacientes con seguimiento inefectivo, 3 (21.5\%) eran rurales, 7 (50\%) urbanos y $4(28.5 \%)$ de Temuco, por lo que la procedencia no mostró una asociación estadísticamente significativa comparada con la procedencia del grupo total de 53 casos p $>0.99$, test exacto de Fisher); en este mismo grupo de 14 pacientes, 13 (92.8\%) tenían cardiopatías de complejidad moderada y alta, y ellos tenían domicilio urbano en el $87.5 \%$ de los casos. La complejidad moderada-alta fue el único factor estadísticamente significativo en el seguimiento inefectivo en CA, comparada con el número de pacientes con cardiopatía de complejidad moderada-alta del total de los 53 pacientes

Tabla 2:

Efectividad de los controles en Cardiología Adultos.

\begin{tabular}{|c|c|c|}
\hline & $\mathrm{N}^{\circ}$ Pacientes & $\%$ \\
\hline Control al día & 9 & 32,1 \\
\hline Control antes de plazo & 1 & 3,6 \\
\hline Control incompleto & 5 & 17,9 \\
\hline Pacientes perdidos & 9 & 32,1 \\
\hline Control pendiente dentro de plazo & 4 & 14,3 \\
\hline Total & 28 & 100 \\
\hline
\end{tabular}




\begin{tabular}{|c|c|c|}
\hline \multicolumn{3}{|c|}{$\begin{array}{l}\text { Tabla 3: } \\
\text { Pacientes con control inefectivo (incompleto o perdidos) en } \\
\text { Cardiología Adultos, según complejidad de la cardiopatía. }\end{array}$} \\
\hline Complejidad & $\mathrm{N}^{\circ}$ Pacientes & $\%$ \\
\hline Leve & 1 & 7,2 \\
\hline Moderada & 5 & 35,7 \\
\hline Alta & 8 & 57,1 \\
\hline Total & 14 & 100 \\
\hline
\end{tabular}

$(p<0.001$, test exacto de Fischer $)$

traspasados ( $\mathrm{p}<0.001$, test exacto de Fisher) (Tabla 3 ).

\section{Discusión:}

La transferencia de CP a CA fue inefectiva en el 22.6\%, y la complejidad de la cardiopatía no fue determinante en este resultado. Una observación relevante en el estudio fue el $50 \%$ de seguimiento inefectivo de los pacientes, una vez incorporados a la atención de CA. Otro hecho significativo en esta revisión fue que, de los pacientes perdidos en CA, $57.1 \%$ correspondían a cardiopatías de alta complejidad (vs $20.8 \%$ del grupo total) y $92.8 \%$ a mediana y alta complejidad (vs $50.9 \%$ del grupo total), lo que mostró una asociación estadísticamente significativa. La procedencia, incluida la ruralidad, no constituyó una variable estadísticamente significativa en el grupo de transferidos ni en el grupo de seguimiento en CA. Comparado con otros estudios existentes a nivel internacional, nuestras cifras de transferencia inefectiva y de seguimiento inefectivo están dentro del rango informado, que es muy amplio, dado que no existe un criterio universal para definir lo que se califica como pérdida de la atención y brecha o lapso de atención ${ }^{10,11}$. Sería deseable que se unifiquen los criterios mencionados, para que se puedan realizar estudios que resulten comparativos y permitan tomar las medidas más efectivas para mejorar los resultados. La ruralidad, por ende, la lejanía al Centro de Atención, no afectó la transferencia ni el seguimiento en CA; sin embargo, nos falta definir las razones por las cuales los pacientes urbanos y de la propia ciudad de Temuco, no tengan una mayor adherencia a control. Un reporte que analiza la distancia al centro de atención de CA como variable de seguimiento, realizado en Bélgica, no influyó en el resultado y estiman que es debido a que ese país es pequeño y las distancias son cortas ${ }^{12}$, lo cual podría ser comparable a lo que ocurre en las distancias y accesos de atención en nuestra Región.

Respecto de la alta complejidad de la Cardiopatía, que fue una variable de resultado muy significativo en la falla de seguimiento una vez ingresados a $\mathrm{CA}$, hay reportes que avalan que estas son un factor protector para la pérdida de seguimiento, pero hay otros que no encuentran tal asociación ${ }^{10,11}$.

En general, se ha estimado como factores de éxito de la transferencia el sexo femenino, cirugías cardíacas previas, cumplimiento de profilaxis antibiótica, buen acceso a la atención, contar con seguros médicos, centros de atención de CP y CA en un mismo edificio y con registros médicos compartidos, conocimiento del paciente de que el seguimiento es necesario, evitar conductas de riesgo vital, mayor edad al último control en $\mathrm{CP}$, transferencia realizada por Cardiólogo Infantil. Además, es positivo que el equipo médico, paciente y padres se centren en la educación y preparación para la transferencia ${ }^{10,11}$. En los estudios internacionales se ha evidenciado la labor de enfermería en el éxito de los procesos de transición. Sin embargo, en nuestro Centro no contamos con este recurso por lo cual los resultados actuales están basados en las labores que se han planificado sólo desde el ámbito médico y administrativo.

Estos hallazgos nos permitieron tomar medidas de mejoría, tanto en la transferencia, como en los controles en $\mathrm{CA}$, que esperamos mejoren los resultados futuros.

Dentro de las medidas locales tomadas se consideraron:

- Aumentar las horas de atención para CC del Adulto de 1 a 4 por semana.

- La atención de CC en adultos se hará solo con Cardiólogo entrenado en esa área.

- Resguardar las horas para la transferencia a CA a través de admisión, con interconsulta en 3 copias (paciente, admisión y ficha clínica).

- Uso de carnet identificador de atención en Policlínico de CC del Adulto con timbre, nombre y diagnóstico para que los pacientes sean fácilmente reconocidos por los funcionarios que tramitan las horas de control y sean ingresados con sus horas resguardadas.

- Informar a los pacientes que, si tienen dificultades en conseguir las horas de CA, recurran a CP para agilizar el proceso.

- Ubicar a los pacientes perdidos para conocer las causas de su falta de control y conseguirles las horas para reincorporarlos. 
- Hacer un "check list" de transferencia con medidas educativas a asumir por el paciente en forma progresiva respecto de su cardiopatía, uso y riesgos de sus medicamentos, riesgos futuros, tratamientos pendientes, calidad de vida, capacidad laboral y deportiva, fertilidad-anticoncepción-riesgos de embarazo y sobrevida, de acuerdo con un modelo de atención del adulto. Dejar una copia en la ficha clínica y entregar otra al paciente para evitar olvidos.
- Aumentar el entrenamiento de Cardiólogos de Adultos en manejo de CC y realización de evaluaciones conjuntas con $\mathrm{CP}$ en pacientes de moderada y alta complejidad.

- Establecer pasada formativa de Becados de CA durante al menos 1 mes en $\mathrm{CP}$ o Centro de $\mathrm{CC}$ del Adulto.

- Aumentar la dotación de Cardiólogos Adultos con formación en CC.

\section{Referencias}

1. SABLE C, FOSTER E, UZARK K, BJORNSEN K, CANOBBIO M, CONNOLLY H, GRAHAM T, ET COLS. Best practices in managing transition to adulthood for adolescents with congenital heart disease: the transition process and medical and psychosocial issues. A scientific statement from the AHA. Circulation. 2011; 123:1454-1485.

2. KNAUTH A, BOSCO V, TONG E, FERNANDES S, SAIDI A. Transition and transfer from pediatric to adult care of young adults with complex congenital heart disease. Current Cardiology Reports 2009; 11: 291-297.

3. BRICKNER E, HILLIS D, LANGE R. Congenital heart disease in adults. First of two parts. The New England Journal of Medicine 2000; 4:256-263.

4. KHAIRY P, IONESCU-ITTU R, MACKIE A, ABRAHAMOWICS M, PILOTE L, MARELLI A. Changing mortality in congenital heart disease. JACC (2010) Vol 56, N 14: 11491157.

5. MOONS P, BOVIJN L, BUDTS W, BELMANS A, GEWILLIG M. Temporal trends in survival to adulthood among patients born with congenital heart disease from 1970 to 1992 in Belgium. Circulation 2010; 122: 2264-2272.

6. GOOSSENS E, STEPHANI I, HILDERSON D, GEWILLIG M, BUDTS W, VAN DEYK K, MOONS P. Transfer of adolescents with congenital heart disease from pediatrics cardiology to adult health care. JACC 2011; 57: 2368-2374.

7. KOVACS A.H., MCCRINDLE B. So hard to say goodbye: transition from paediatric to adult cardiology care. Nat. Rev. Cardiol. 2014; 11:51-62.

8. MARELLI A, IONESCU-ITTU, R, MACKIE A, GUO L, DENDUKURI N, KAOUACHE M. Lifetime prevalence of congenital heart disease in the general population from 20002010. Circulation 2014; 130: 749- 756.

9. WEBB G, WILliAMS R. 32nd Bethesta Conference: Care of the adult with congenital heart disease. JACC 2001; 37: 1161-98.

10. HEERY E, SHEEHAN A, WHILE A, COYNE I. Experience and outcomes of transition from pediatric to adult health care services for young people with congenital heart disease: a systematic review. Congenit Heart Dis 2015; 10: 413-427.

11. STEWART K, CHAHAL N, KOVACS A, MANLHIOT C, JELEN A, COLLINS T, MANLHIOT C, et cols. Readiness for transition to adult health care for young adolescents with heart disease. PediatrCardiol 2017; 38:778-786.

12. GOOSSENS E, STEPHANI I, HILDERSON D, GEWILLIG M, BUDTS W, VAN DEYK K, MOONS P. Transfer of adolescents with congenital heart disease from pediatric cardiology to adult health care. JACC 2011; 57:2368-2374. 